\title{
Abscess or Tumor? When a Retroperitoneal Mass on Computerized Tomography Turns Out to Be a Rare Soft Tissue Growth
}

\author{
Ilya Noginskiy Neil Nimkar Madhumati R. Kalavar \\ New York Presbyterian Brooklyn Methodist Hospital, Brooklyn, NY, USA
}

\section{Keywords}

Desmoid fibromatosis · Soft tissue tumor · Retroperitoneal mass · Abscess · Tamoxifen

\begin{abstract}
A retroperitoneal finding on a computerized tomography scan, in a symptomatic patient, can harbor the clinician to many differential diagnoses from infectious to malignancy. Desmoid fibromatosis (DF), a relatively innocuous mass that can spread locally, can be found in that anatomical region. Even for a rare tumor such as DF, our patient did not meet the usual benchmarks of this cancer, being an elderly female and having it appear as an abscess radiologically. Timely clinical response with radiotherapy and tamoxifen allowed our patient's DF to regress and resolved her symptoms.
\end{abstract}

\section{Introduction}

Desmoid-type fibromatosis (DF) is a rare sarcomatous tumor subtype, which arises from mesenchymal cells, and is locally invasive [1]. DF is known to be indolent in nature but may cause symptoms and complications when it is intra-abdominal. It usually presents in those between the ages of 30 and 40. It can present as an intra-abdominal abscess or even a fistula, and later upon pathologic investigation is found to be DF. Therefore, there are various presentations of this rare tumor, one of which is being presented here along with its treatment options. 

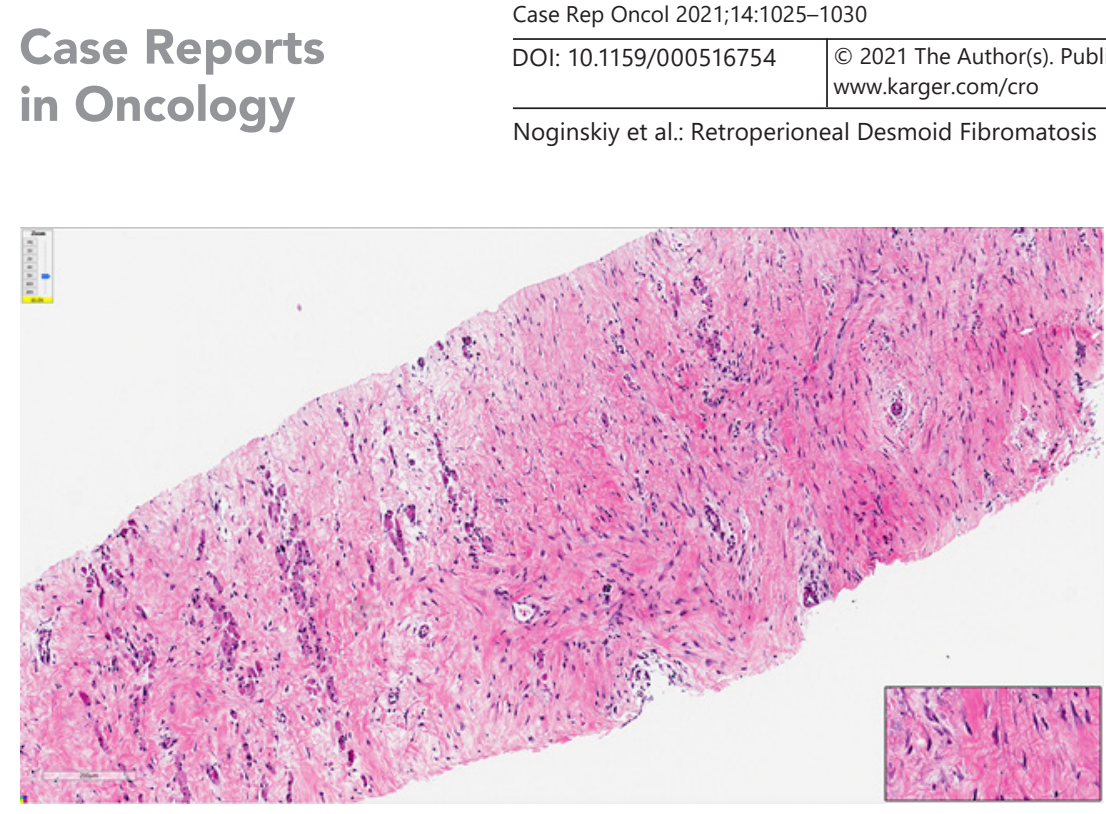

Fig. 1. Right iliopsoas mass core biopsy at low power field, $\times 5$ (H\&E stain).

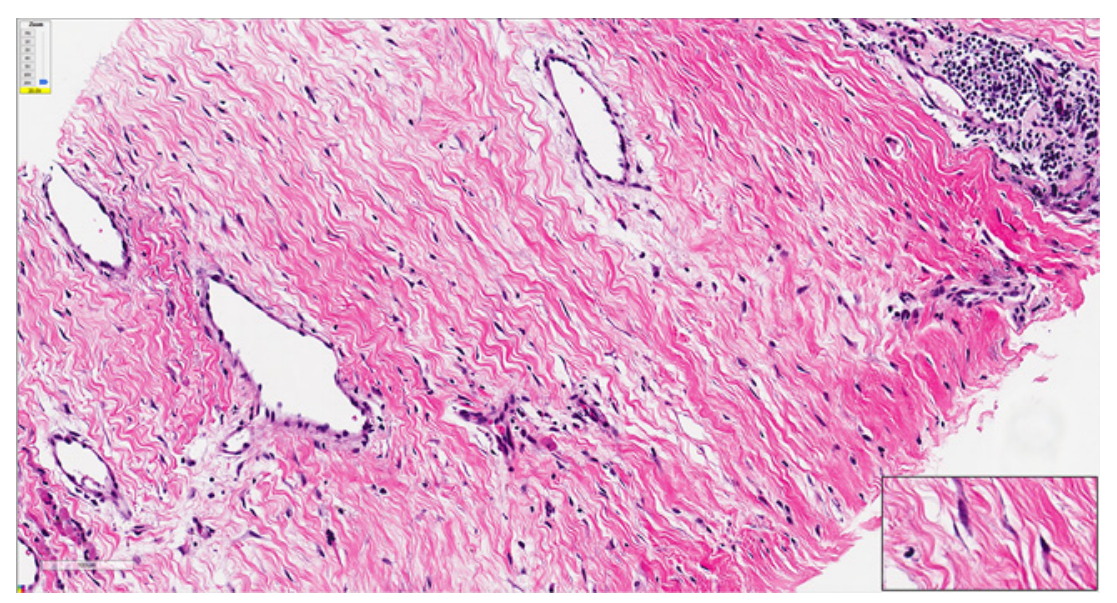

Fig. 2. Photomicrograph high power field, $\times 20$ (H\&E stain).

\section{Case Presentation}

This is a case of an 83-year-old female initially presenting with right-sided flank and abdominal pain for a duration of 3 months along with a small lump on her right mid-back area, which increased in size over the next 2 weeks. Upon imaging, the patient was found to have an extensive multiloculated perinephric abscess with invasion of right iliopsoas muscle and an adjacent mass along with bilateral pleural effusions. Her labs at the time of diagnosis were suggestive of microcytic anemia with a hemoglobin of 8.6 and a Mean Corpuscular volume of 78 with no other abnormalities. The patient initially underwent a computerized tomographyguided drainage of the abscess along with IV antibiotics and had a biopsy of the mass which showed a skeletal muscle-derived tumor further characterized as a retroperitoneal (RP) desmoid-type fibromatosis with invasion into the skeletal muscle (Fig. 1-3). Based on the imaging (Fig. 4), the tumor was staged as a T4N0M0. Further immunostaining was done showing B-Catenin positivity and very low labeling index for KI-67. The patient was then evaluated by radiation oncology and completed radiation therapy with 37 fractions for a total of 6,600 Gy. The patient was subsequently treated with daily tamoxifen ( $20 \mathrm{mg}$ daily) and

\section{Karger'k}




\section{Case Reports in Oncology}

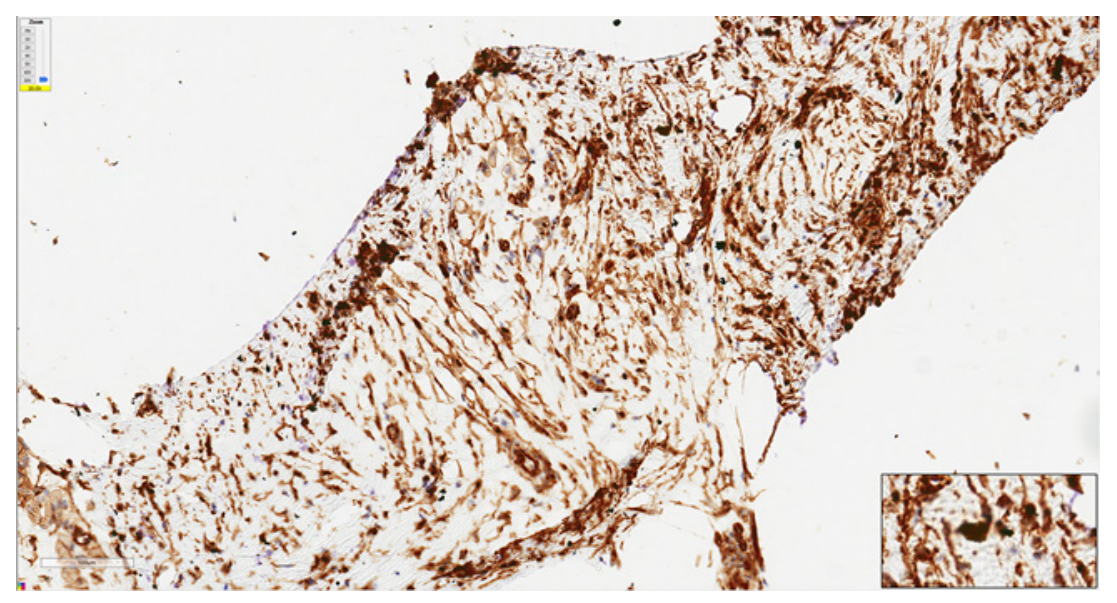

Fig. 3. Photomicrograph high power field, $\times 20$ (IHC stain; tumor cells positive for B-Catenin). IHC, immunostaining.

Fig. 4. Right RP mass shown on CT. RP, retroperitoneal; CT, computerized tomography.

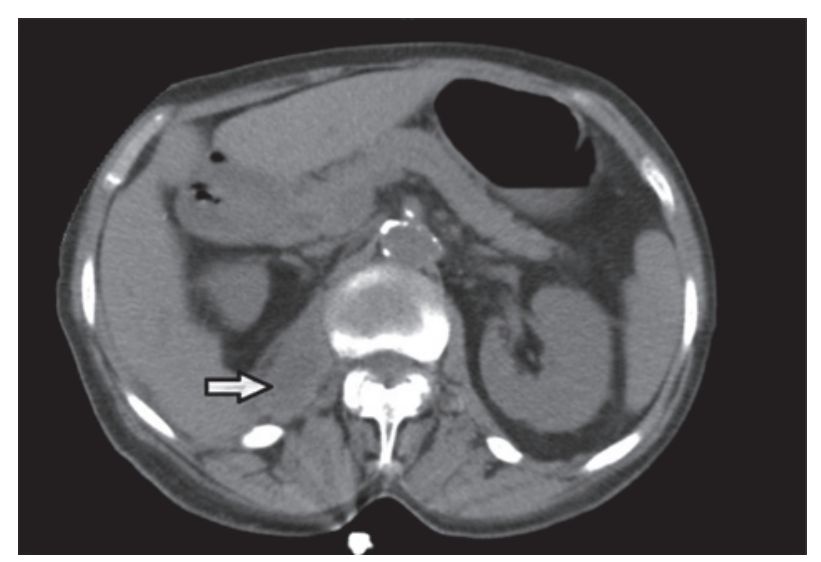

upon repeating magnetic resonance imaging of the abdomen/pelvis there was resolution of the initial RP mass.

\section{Discussion}

Desmoid fibromatosis (DF) is a benign but locally extending tumor that arises from cells of mesenchymal origin [1]. Its major regional subtypes consist of tumors located in the abdominal cavity, abdominal wall, or the extremities/trunk [1,2]. Genetically, heritable DF is associated with the abdominal cavity variant, while the sporadic version is associated with the abdominal wall and musculoskeletal variants [1]. These tumors do not typically metastasize but can cause local invasion and pain from mass effect (as in our case). Worldwide epidemiological data on the prevalence of DF is limited, but population-based studies in the USA and Finland report the condition to be present in about 2-5 in 1 million people $[3,4]$.

There is a significant correlation with DF and familial adenomatous polyposis (FAP); a meta-analysis of 4,625 FAP patients showed that 12\% developed DF [5]. The APC gene is associated with DF progression in those with FAP. Abdominal surgery contributed to DF development in those with FAP as well [6]. The sporadic DF type is associated with B-Catenin 
deregulation. A 2008 study showed that CTNNB1 aberrations (resolving in the overly active WNT1/B-catenin pathway), particularly 3 mutations in 2 codons of exon 3, were associated with $85 \%$ of desmoids [7]. DF is also noted to be estrogen respondent, with estrogen receptor (ER) antagonism being one of the mainstays of therapy for decades. In a study looking at 40 cell lines of extra-abdominal fibromatosis, ER Beta was expressed in 100\% of the samples (not ER Alpha) [8]. Apart from the more prolific CTNNB1 and ER-driven influence on DF, mutations in JAK/STAT, Notch, PI3 kinase/AKT, mTOR, and hedgehog have all been implicated in the cancer's development. Each of these pathways currently has targeted therapy undergoing trials [9].

Our case is unusual in presentation since it is a rare tumor, found in an atypical location, is of sporadic origin, and seen in a patient whose age is out of the typical range for DF. The tumor resembled an abscess in the RP space on initial computerized tomography scan. Desmoid tumors constitute $<1 \%$ of RP tumors [10]. In one retrospective study of 25 patients with abdominal DF, only $9 \%$ were found to be in the retroperitoneum [11]. In our patient, core biopsy of the right ileo-psoas mass showed a fibromuscular lesion with significant atypia, with atrophy of trapped skeletal muscle fibers, and focal chronic inflammation (few foci) with lymphoplasmacytic and histiocytic infiltration.

There are only a couple of cases of RP DF that have been reported with an inflammatory/ abscess forming in the muscle. One was in a 43-year-old female, originating between the left iliocostalis and psoas muscles, showing paraspinal muscle atrophy around the tumor [12]. The other, in a 35-year-old male with a mass in the anterior bladder wall with histology showing purulent inflammation of local adipose tissue and chronic lymphadenitis. In both of these cases, the DF was surgically removed, whereas our patient was treated with radiation and hormonal therapy [13]. More reports of abscess formations in desmoid tumors are seen in the intra-abdominal variants that are mesenteric, but scant evidence exists for abscesses forming in the retroperitoneum [14].

There is no defined treatment protocol in DF and cases are managed on an individual basis and on clinical symptomatology. Generally, the first tactic in treatment is a "wait and watch" approach, in cases of asymptomatic DF. In a review of 31 articles of patients treated for DF, the control rate for patients treated with an observational approach compared favorably in management with surgery and resulted in disease control rates between 60 and 92\% [15]. Surgery or radiotherapy is considered when the tumor does not regress spontaneously with observation. Local control rates after complete surgical resection are $\sim 80 \%$ at 5 years with abdominal DF having the best prognosis. In those where surgery is not an option, radiotherapy to a dose of 56 Gy in 28 once-daily fractions of 2 Gy has been shown to provide adequate local control in the majority of progressive patients [16].

Estrogen has been observed to be a driving proliferator of DF as ER-B receptors are overexpressed. From pharmacological therapy, tamoxifen has been most extensively studied and has been traditionally used for tumor regression/complete resolution. Raloxifene has been trialed as well with a favorable response. Blocking estrogen's downstream effect blocks the cell's survival. The WNT/B-Catenin pathway that has an established role in DF developments, results in COX enzyme products that later lead to autocrine-driven proliferation. In light of this, nonsteroidal anti-inflammatory drugs such as sulindac, are used to target the downstream effects of the WNT pathway. Nonsteroidal anti-inflammatory drugs were studied in combination of hormone modulators (tamoxifen), as well as solo therapy. When hormonal therapies fail or tumors are aggressively growing, chemotherapy such as methotrexate and/or vinblastine/ vinorelbine can be used at low doses. Imatinib, sunitinib, and sorafenib are being trialed to target the tyrosine kinase receptor, another proliferative player in DF, with mostly stabilizing, rather than resolving rates. Additionally, ongoing trials are exploring downstream effectors of the JAK/STAT, Notch, PI3 kinase/AKT, mTOR, and hedgehog pathways $[9,16]$.

\section{Karger'}


Our patient presented with RP DF with an iliopsoas biopsy showing spindle cell proliferation with trapped atrophied skeletal muscle fibers, later reported as "adjacent abscess formation." This is a rare presentation of DF, an addition to only a few case reports of RP skeletal muscle involvement of the sporadic variant. Although it is known that tumors can cause abscesses by blocking vascular supply to the tissue, this is the first such report with skeletal muscle DF in the retroperitoneum.

This case report should serve as an example that large RP masses must be explored more judiciously. This patient had a presentation to her primary care physician with back/flank pain and a mass abutting out of her skin seen on examination of her back. The mass was rapidly growing and was causing the patient distress. Timely and careful radiological and histological assessment is very important in this setting. In RP DF, neurovascular structures (i.e., autonomic plexi, ganglii, and branches of aorta/Vena cava) can be compromised if the rate of tumor spread is not taken seriously by the clinician. Abscess formation of a large mass, because of compromised blood supply, is another potentially morbid outcome seen in this report as well. Fortunately, this patient had an expert team of physicians and the patient did not suffer consequences from the abscess itself; her tumor resolved with radiation therapy and adjuvant hormonal therapy with tamoxifen.

\section{Acknowledgments}

Special thanks to the radiation oncology department at NYP Brooklyn Methodist Hospital for their role in this patient's recovery. Much appreciation goes to the Interventional Radiology Department at NYP Brooklyn Methodist Hospital and the tissue pathologists at NYP Weill Cornell Hospital NYC for their significant roles in diagnosing this patient's condition.

\section{Statement of Ethics}

The subject of this case report has given their written informed consent to be published. This includes their medical history, images, and biopsy slides.

\section{Conflict of Interest Statement}

We report no conflict of interest in this case report.

\section{Funding Sources}

We report no sponsors of funding of this case report.

\section{Author Contributions}

I.N. has gathered the relevant scientific and published information on the subject matter and has written the "abstract" and "discussion" portions of the case report. N.N. has been involved with treatment of the patient and has written the "introduction" and "case presentation" portions of the case report. M.K. is the patient's primary treating physician and has provided the assessment, treatment plan, and follows for her. She was directly involved in the analysis for this paper and has supervised its content. 


\section{References}

1 Fletcher CDM, Bridge JA, Hogendoorn PCW, Mertens F. WHO classification of tumours of soft tissue and bone. Lyon: IARC; 2013.

2 Amanda KA, Joseph Kim MD. Diseases of the peritoneum and retroperitoneum. gastrointestinal tract and abdomen; 2014. Available from: https://www.stonybrookmedicine.edu/sites/default/files/acs_surgery_ hipec.pdf.

3 Fallen T, Wilson M, Morlan B, Lindor NM. Desmoid tumors: a characterization of patients seen at Mayo Clinic 1976-1999. Fam Cancer. 2006;5:191-4.

4 Reitamo JJ, Häyry P, Nykyri E, Saxén E. The desmoid tumor. I. Incidence, sex-, age- and anatomical distribution in the Finnish population. Am J Clin Pathol. 1982;77:665.

5 Sinha A, Tekkis PP, Gibbons DC, Phillip RK, Clark SK. Risk factors predicting desmoid occurrence in patients with familial adenomatous polyposis: a meta-analysis. Colorectal Disease. 2011;13:1.

6 Nieuwenhuis MH, De Vos Tot Nederveen Cappel W, Botma A, Nagengast FM, Kleibeuker JH, Mathus-Vliegen EM, et al. Desmoid tumors in a dutch cohort of patients with familial adenomatous polyposis. Clin Gastroenterol Hepatol. 2008;6:2.

7 Lazar AJ, Tuvin D, Hajibashi S, Habeeb S, Bolshakov S, Mayordomo-Aranda E, et al. Specific mutations in the beta-catenin gene (CTNNB1) correlate with local recurrence in sporadic desmoid tumors. Am J Pathol. 2008; 173:1518.

8 Deyrup AT, Tretiakova M, Montag AG. Estrogen receptor-beta expression in extraabdominal fibromatoses: an analysis of 40 cases. Cancer. 2005;106:208.

9 Timbergen MJM, Smits R, Grünhagen DJ, Verhoef C, Sleijfer S, Wiemer EAC. Activated signaling pathways and targeted therapies in desmoid-type fibromatosis: a literature review. Front Oncol. 2019;9:397.

10 Rajiah P, Sinha R, Cuevas C, Dubinsky TJ, Bush WH Jr, Kolokythas O. Imaging of uncommon retroperitoneal masses. Radiographics. 2011;31:949.

11 Einstein DM, Tagliabue JR, Desai RK. Abdominal desmoids: CT findings in 25 patients. AJR Am J Roentgenol. 1991;157:275.

12 Kikkawa A, Kido A, Kumai T, Hoshida T. Extraabdominal fibromatosis in retroperitoneal space. World J Surg Oncol. 2004;2:33.

13 Campara Z, Spasic A, Aleksic P, Milev B. An aggressive retroperitoneal fibromatosis. Med Arch. 2016;70:154.

14 Alemanno G, Zambonin D, Sturiale A, Cavalli T, Bellucci F, Pesi B, et al. A multidisciplinary approach to desmoid tumors. When intra-abdominal fibromatosis degenerates into an abscess, which is the right treatment? Int J Surg Case Rep. 2013;4:757.

15 Penel N, Le Cesne A, Bonvalot S, Giraud A, Bompas E, Rios M, et al. Surgical versus non-surgical approach in primary desmoid-type fibromatosis patients: a nationwide prospective cohort from the French sarcoma group. Eur J Cancer. 2017;83:125-31.

16 Kasper B, Baumgarten C, Garcia J, Bonvalot S, Haas R, Haller F, et al. An update on the management of sporadic desmoid-type fibromatosis: a European consensus initiative between sarcoma patients euronet (SPAEN) and European organization for research and treatment of cancer (EORTC)/soft tissue and bone sarcoma group (STBSG). Ann Oncol. 2017;28:2399. 